\title{
Effects of Moisture on Mechanical and Piezoresistive Properties of Cement Composites containing Carbon Fiber during long age
}

\author{
Wangyanfeng ${ }^{1,2, a^{*}}$, Zhaoxiaohua $^{1, \mathrm{~b}}$, Zhaoyi $^{2, \mathrm{c}}$, Bianyadong $^{2, \mathrm{~d}}$ \\ 1. Department of civil engineering, Shantou University, Shantou, 515063 \\ 2. Department of Civil Engineering and Architecture, Zhongyuan University of Technology, Zhengzhou, 450007
}

\begin{abstract}
This paper presents a compressive test program designed to determine the mechanical and self-sensing properties of cement-matrix composites containing carbon fiber. Two kinds of mixes with $0.5 \%$ and $0.75 \%$ carbon fiber (CF) were prepared. The mechanical and piezoresistive properties of the cement-matrix composites were evaluated at 28, 90, 180, 270, and $360 \mathrm{~d}$. The results show that the mechanical properties were enhanced in most cases. However, the compressive strength of carbon fiber reinforced cement-matrix composites decreased compared with the reference mix at the early curing ages, which agrees with the results mentioned in the literature. What is worth to mention, the $360 \mathrm{~d}$ compressive strength of carbon fiber reinforced cement-matrix composites reached the same level as that of the reference. Moreover, both negative and positive piezoresistivity were observed during the experiments. The amplitude of piezoresistivity was found to change with the variation of moisture content, and was not directly proportional to the magnitude of the stress. In the elastic stage with smaller stress amplitude, the piezoresistivity amplitude was larger. When the stress amplitude was multiplied, the piezoresistivity change was not synchronous. The gauge factor for the composite with $0.75 \% \mathrm{CF}$ was higher than that of the composite with $0.5 \% \mathrm{CF}$ and commercially available strain gauges.
\end{abstract}

\section{Introduction}

Recently, a new type of self-sensing material, carbon fiber reinforced cement-based composites with piezoresistivity, has shown promise for long-term health monitoring owing to the advantages of durability, low cost, and compatibility with a cement matrix [1-6]. Piezoresistivity refers to a coupled electrical-mechanical property that the electric resistivity of a material changes with strain. If the electric resistivity decreases with compressive strain, piezoresistivity is defined to be positive. Otherwise, piezoresistivity is negative. With this property, the cementitious composites containing CF are able to sense strain through the variation of electric resistance.

This finding is of great importance for the nondestructive monitoring of concrete structures as the materials themselves can be used as stress/strain sensors to monitor both the generation and propagation of damage. Thus, real-time monitoring of concrete structures can be achieved simply by measuring the electrical resistance, thus enabling timely repair or other hazard-precaution measures. Professor Chung [1] first proposed that a proper volume concentration of carbon fiber forms a conductive network in concrete, and the resistivity of this mix clearly changes when strain is applied. Since then, cement-based composites with

"Corresponding author: ${ }^{\mathrm{a}} 6706 @$ zut.edu.cn;

bxhzhao2019@163.com; 'Zhaoyi091218@163.com; dbyd@zut.edu.cn; piezoresistivity have attracted much attention [7-11].

However, the cementitious composites containing CFs are porous materials, and capillary water exists in cement matrix even if the hydration process has completed. Moreover, the cementitious composites usually work in a moisture condition. One concern then arises that the piezoresistivity may be affected by moisture content in the composites, as water provides ions that contribute to ionic conduction. This effect is relevant to the practical application of the composites as piezoresistive structural materials. Although there has been a report on the effect of moisture on the electrical conductivity and dielectric behaviour [12-14]. Few studies have focused on the effect of moisture on the piezoresistive behavior. Zhao et al [14] studied the effect of MC on the piezoresistivity by drying, the results show that the relative change in resistance varies with the moisture content of the composites. When the moisture is higher, the piezoresistivity is positive. While the moisture content is reduced after specimens' being oven dried, the piezoresistivity is negative. However, What is worth mentioned, Drying can change the microstructure of cement matrix, especially the shrinkage deformation. DDL et al [15] conducted the experiments about the MC on piezoresistivity, the results give the higher and lower cases, the worst scenario is associated with both the extreme moisture content corresponding to water saturation and a fiber content below the percolation 
threshold. For this worst scenario, moisture diminishes the piezoresistivity. The corresponding state after drying at room temperature are compared in terms of the sensitivity, accuracy, and repeatability of the piezoresistivity based strain sensing. They inferred the intermediate states is between the above states. Yet the influence of MC on piezoresistivity has not been fully elucidated, especially the MC in practice.

This paper is focused on investigating the effect of moisture (free water due to water absorption after curing) on the mechanical properties and the piezoresistivity of carbon fiber-reinforced mortar. The investigation involves comparison of the changes of mechanical properties and the piezoresistivity at different ages. States with water content higher than that of the dry state and lower than that of the wet state are addressed, because such intermediate states are more commonly encountered in practice.

Table 1. Composition and physical properties of cement

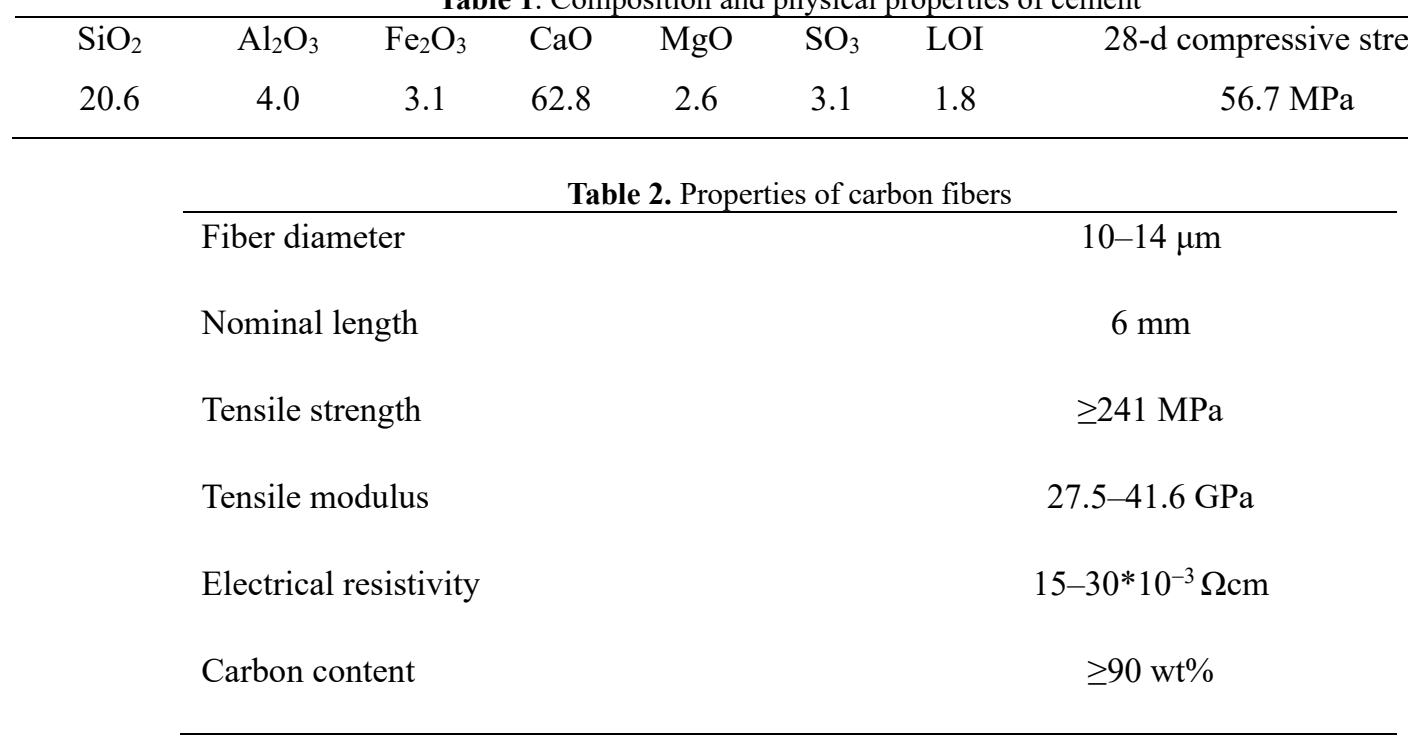

Three types of mortar were designed for the testing: plain cement mortar (CM) and carbon-fiber-reinforced cement mortar (CFRCM). The details are listed in Table 3.

A rotary mixer with a flat beater was used for mixing. In the case of CM, cement, sand, water, and deformer were added and mixed in the rotary mixer for $\sim 5 \mathrm{~min}$. In the case of CFRCM, methylcellulose was first dissolved in water, and then the carbon fibers and deformer were added. The methylcellulose solution was stirred till the fibers completely dispersed. Then, the solution, cement, and sand were mixed in the rotary mixer for $\sim 5 \mathrm{~min}$.

\section{Experimental Methods}

\subsection{Materials}

The cementitious material used in this study is Portland cement. Its chemical composition and physical properties are shown in Table 1. The fine aggregate was natural sand with a specific gravity of $2.50,100 \%$ of which passed a 2.36-mm sieve.

The carbon fibers are isotropic pitch based, unsized, and had a nominal length of $6 \mathrm{~mm}$ and diameter of 10-14 $\mu \mathrm{m}$; they were obtained from East-Asia Company, Shengyang, China. The properties of carbon fibers are shown in Table 2. Methylcellulose was used to disperse the carbon fibers. A deformer was also added.
After pouring the mix into oiled molds $(40 \times 40 \times$ $160 \mathrm{~mm}^{3}$ and $70.7 \times 70.7 \times 70.7 \mathrm{~mm}^{3}$ ), an electric vibrator was used to facilitate compaction and reduce the amount of air bubbles.

Eleven specimens were prepared for each mix. Three specimens $\left(40 \times 40 \times 160 \mathrm{~mm}^{3}\right)$ were used for flexural testing, and three specimens $\left(70.7 \times 70.7 \times 70.7 \mathrm{~mm}^{3}\right)$ were used for compressive testing. Five specimens $(40 \times$ $40 \times 160 \mathrm{~mm}^{3}$ ) were used for pressure-sensitive testing. 

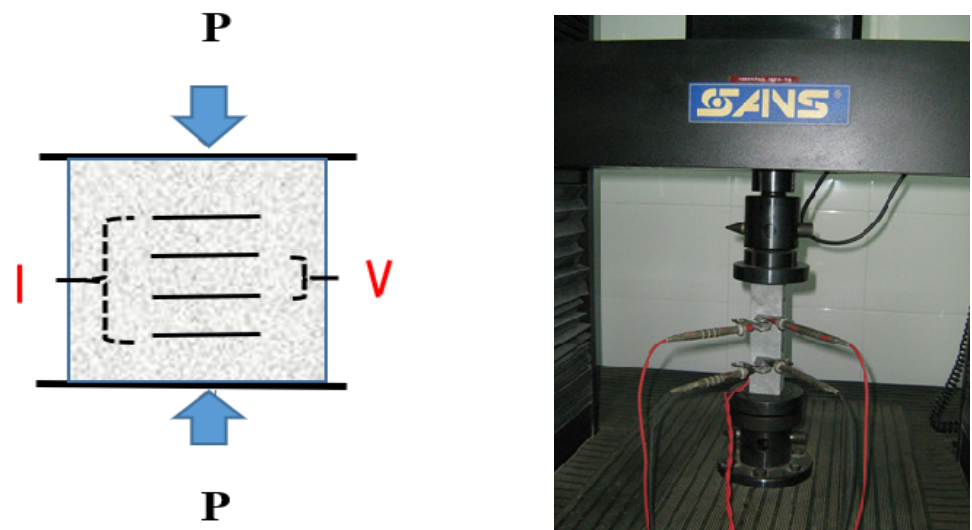

Fig.1 Sketch map for electrode arrangement and direct-current four probe method

During the casting of these specimens for pressure-sensitive testing, four stainless steel sheets were embedded in each of them to measure electrical resistance using the four-probe method, which is shown in fig.1. The two outer sheets $(100 \mathrm{~mm}$ apart, symmetrically positioned relative to the center plane perpendicular to the longitudinal direction) were used for passing the current, while the inner two sheets $(60 \mathrm{~mm}$ apart, symmetrically positioned relative to the center plane perpendicular to the longitudinal direction) were used for measuring the voltage.

All the specimens were demolded after $1 \mathrm{~d}$. Then, they were cured in a saturated calcium carbonate solution with a constant temperature of $25{ }^{\circ} \mathrm{C}$. Twenty-eight days later, they were removed from the saturated calcium carbonate solution. The first group (including eight mixes) was tested. Other groups were tested on $90 \mathrm{~d}, 180 \mathrm{~d}, 270$ $\mathrm{d}$, and $360 \mathrm{~d}$. During this time, the specimens were naturally cured in the room where the temperature and moisture were changing with the time. We record the values of the week before and after the test and take the mean as the representative value of the period. The variation of mean temperature and humidity with age was shown in Fig. 4.

Table 3. Mixes of cement-matrix composites (by weight; $\mathrm{CF}=$ carbon fibers)

\begin{tabular}{llcccccc}
\hline Mixes & No. & Water & Cement & Sand & $\begin{array}{c}\text { Carbon } \\
\text { fibers }\end{array}$ & Dispersant & Defoamer \\
\hline $\begin{array}{l}\text { Plain } \\
\text { Plain }+0.5 \%\end{array}$ & CM & 0.5 & 1 & 1.5 & & & $0.13 \%$ \\
CF & CFRCM1 & 0.5 & 1 & 1.5 & $0.50 \%$ & $0.35 \%$ & $0.13 \%$ \\
$\begin{array}{l}\text { Plain }+0.75 \% \\
\text { CF }\end{array}$ & CFRCM2 & 0.5 & 1 & 1.5 & $0.75 \%$ & $0.53 \%$ & $0.13 \%$ \\
\hline
\end{tabular}

\subsection{Testing}

At 28 d, 90 d, 180 d, 270 d, and 360 d, flexural and compressive strengths of the mixes were tested. The flexural strength of specimens was tested using the three-point bending method in universal testing machine at a cross head speed of $0.5 \mathrm{~mm} / \mathrm{min}$ until failure occurred. The compressive strength was tested using a SANS testing system in displace-controlled mode with a displacement rate $1.0 \mathrm{~mm} / \mathrm{min}$. For pressure-sensitive testing, to evaluate the effect of moisture and stress level, the temperature and moisture content in the lab were tested for seven days before the pressure-sensitive testing, and their average were used in this study. All these specimens were weighed before the testing every time. Then, the specimens were compressed under cyclic loading. The loading was applied on two ends in the longitudinal direction of each specimen with a rate of 0.5 $\mathrm{mm} / \mathrm{min}$. The loading has five levels. The first level is 5 $\mathrm{KN}$, followed by $10 \mathrm{KN}, 15 \mathrm{KN}, 20 \mathrm{KN}$, finally $25 \mathrm{KN}$. The strains were measured using 6-mm long foil gauges attached to the surfaces of the specimens during the loading. Both the strain and load data were acquired using a static data logger. An Agilent 34401A multimeter was to measure the resistance as shown in Fig. 2.

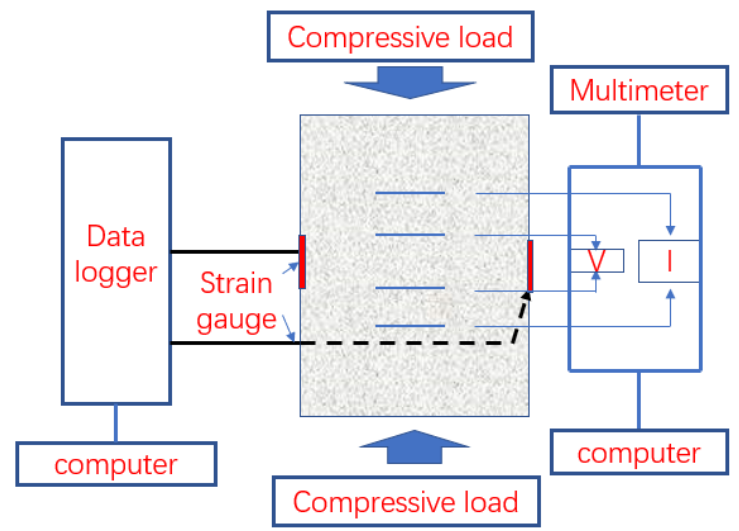

Figure 2. Schematic diagram of measurement of self-sensing properties 


\section{Results and Discussions}

\subsection{Mechanical strength}

Fig. 3(a) shows the changes of flexural compressive strength of carbon fiber reinforced cement mortar with age. The flexural strength of CFRCMs increases with the increase of age. At early age, the flexural strength of carbon fiber cement mortar is slightly higher than that of cement mortar $(\mathrm{CM})$. With the increase of age, the flexural strength of CFRCM is obviously higher than that of CM. At 180 days, the flexural strength of CFRCM1 reaches $11.21 \mathrm{Mpa}$, which is $8.94 \%$ higher than that of $\mathrm{CM}$, and the flexural strength of CFRCM 2 reaches 11.71 Mpa, which is $13.8 \%$ higher than that of CM. At 270 and 360 days of age, the flexural strength of CFRCM1 reached $11.56 \mathrm{Mpa}$ and $11.82 \mathrm{Mpa}$, respectively, which increased by $8.85 \%$ and $10.47 \%$ compared with that of CM. The flexural strength of CFRCM2 is as high as $12.51 \mathrm{Mpa}$ and $12.71 \mathrm{Mpa}$, respectively, which are $17.8 \%$ and $18.79 \%$ higher than that of CM.

As shown in Fig. 3(b), the compressive strength of CFRCM is lower than that of CM when the age is small. This may be due to the addition of carbon fibers, which increases the initial defects of cement mortar, so the compressive strength of CFRCM decreases at early age.
Previous study showed that the addition of carbon filaments in cement matrix would decrease the mechanical properties of cement matrix composites [13-14]. Clearly, present results support this conclusion。 However, paper [11] obtained the higher compressive strength of CFRCM at 3,28 days by using the ultrasonic wave to disperse the fibers. It can be inferred that the effective dispersion of carbon fibers in cement matrix is very beneficial to improve its compressive strength.

With the increase of age, the compressive strength of CFRCM is closer to that of CM. At 270 and 360 days, the compressive strength of CFRCM is almost equal to that of CM. The hydration process of cement paste is relatively long. With the increase of age, the influence of initial defects caused by carbon fiber addition on its compressive strength is weakening. When the content of carbon fiber is not particularly large, the compressive strength of carbon fiber cement-based materials will be close to that of plain cement mortar after a longer age, at the same time, the flexural strength will be higher to that of CM. Alkaline curing may be an important factor, which may weaken air carbonization. Obviously, the mechanical properties of CFRCM1 and CFRCM2 increased obviously with the time going. Similar law of strength development can be found in document [18].

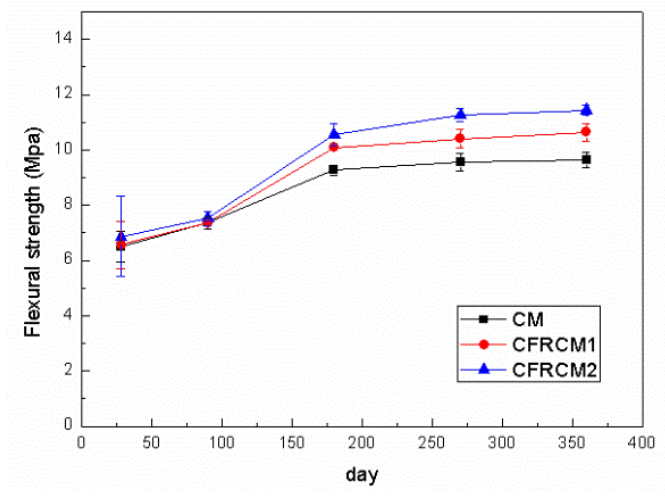

(a) flexural strength of mixes

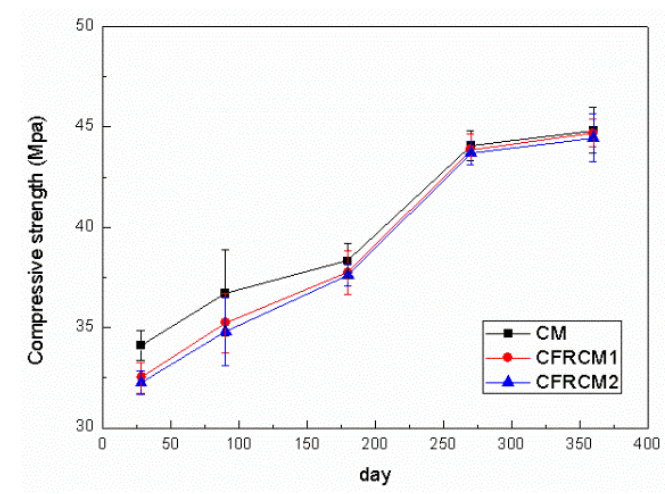

(b) compressive strength of mixes

Figure 3. the results of the mechanical testing

\subsection{Electrical properties}

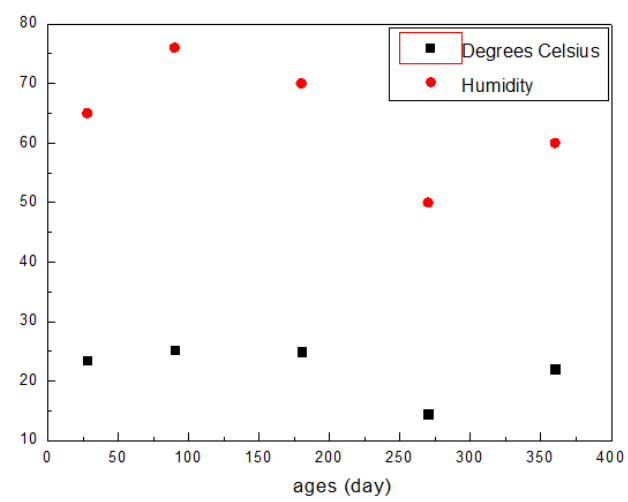

Fig. 4 Changes of humidity and temperature in test room with age

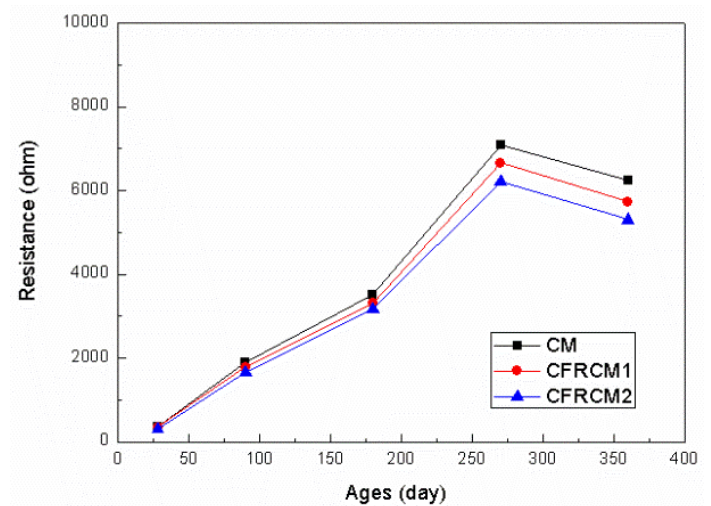

Fig. 5 Variation of composite resistances with age

The hydration of cement is a long process. Hardened cement mortar are composed of hydration products, unhydrated clinker particles, and pore network occupied with water and a small amount of air, forming a porous solid-liquid-gas system. According to the Archie's law 
$[16,17]$, the resistance of a mortar can be used as an index for the water content and connectivity of pores inside a specimen. The resistance of mortar should increase with curing age under our experimental conditions. However, cement mortar is a porous material, which is easily affected by ambient humidity. Figure 5 shows the change in the average temperature and humidity at different ages. The changes in the average mass of mixes are shown in Table.1, a turning point was observed at $270 \mathrm{~d}$. In the later days, the mass of mixes increased with the increase in humidity. The average resistance of mixes showed the opposite trend. The resistances rapidly increased at the early age for $\mathrm{CM}$ and CFRCMs. At more than 90-d curing, the resistances of mixes have similar increasing trends. When the ambient moisture content clearly decreased between $180 \mathrm{~d}$ and 270 d, the lines in Fig. 5 became steeper. The resistances of mixes reached the maximum values at the $270 \mathrm{~d}$ with the lowest humidity. The resistances of mixes decreased when the humidity increased at later days.

The resistance of carbon fiber cement-based materials depends on its internal conductivity network, which can

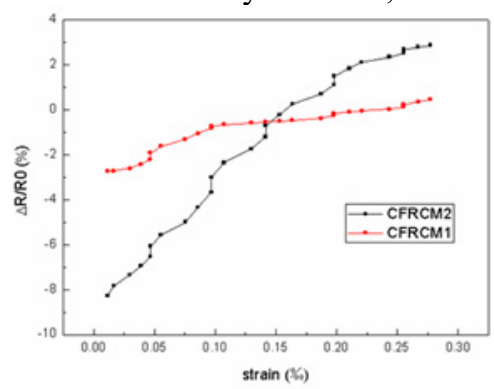

(a) 28 days

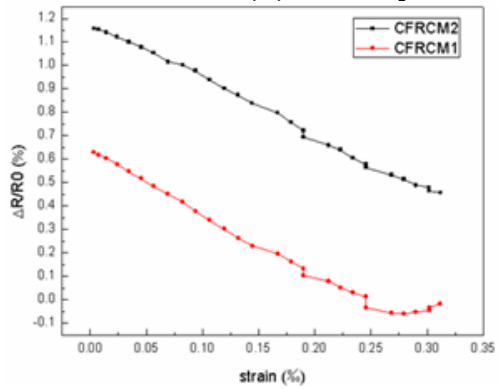

(c) 180 days be roughly divided into three aspects: (1) ionic conductivity of solution, (2) electronic conductivity of carbon fiber, (3) tunneling effect of carbon fiber. The ionic conductivity of the solution depends on the concentration and connectivity of ions. In this paper, it is assumed that the concentration of ions remains unchanged, and the connectivity changes obviously with the increase of age. The older the age, the worse the connectivity and the greater the resistance. On other hand, with the increase of ambient humidity, the water absorbed by cement based material increases, the ionic conductivity increases, and the resistance decreases. On the contrary, the resistance increases.

\subsection{Piezoresistivity of mixes}

In order to compare the piezoresistive effect of CFRCM1 and CFRCM2, the piezoresistive effect curves corresponding to the second loading stage in the second loading cycle of different ages were compared and analyzed as shown in fig.6(a-e).

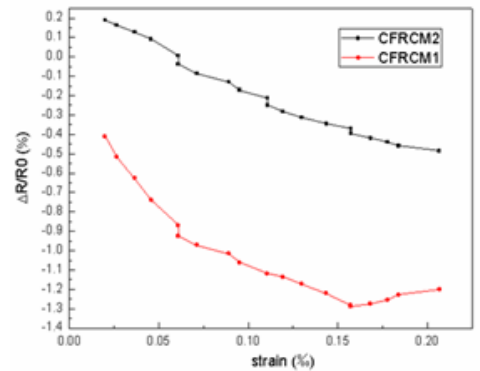

(b) 90 days

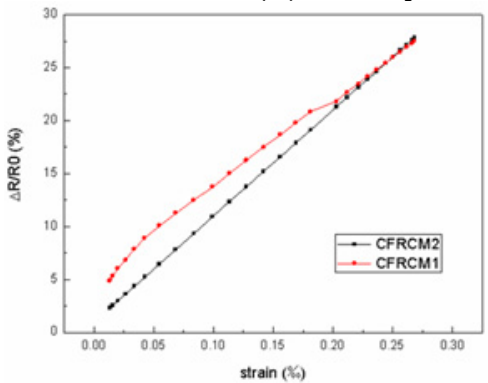

(d) 270 days

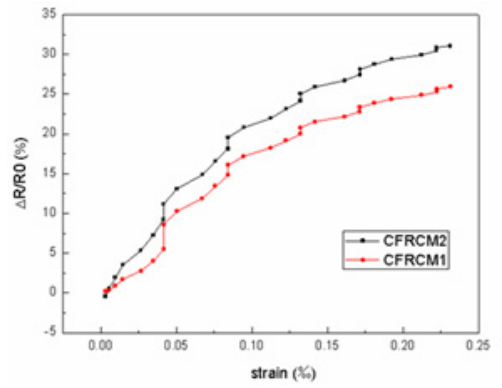

(e) 360 days

Fig.6 Comparison of piezoresistive effect curves at different ages under the same strain level.

As shown in fig.6, when the age is 28 days, the change rate of piezoresistive effect curve of CFRCM2 is significantly higher than that of CFRCM1, which means that CFRCM2 is more sensitive to the change of strain and its resistivity changes greatly. When the age is 90 days, the sensitivity of the two to strain changes decreases. The absolute value of piezoresistive effect curve slope of CFRCM1 is slightly higher than that of CFRCM2, but decreases when the strain is large. When the age is 180 days, their sensitivity to strain is close to 
each other, and changes approximately parallel with the increase of strain. When the age is 270 days and 360 days, the slope of piezoresistive effect curve of CFRCM2 is higher than that of CFRCM1. In conclusion, CFRCM2 has better piezoresistive performance and better linear variation of sensitivity.

In view of the similarity of piezoresistive effect curves of specimens at the same age, only piezoresistive curves of different ages and few stages are given for comparative analysis. The piezoresistive effect curves of CFRCM2 at different ages are shown in Fig. 7-11. In order to compare the sensitivity of piezoresistive effect curves in different age periods, the test values corresponding to the second loading and unloading cycle in the second loading cycle were analyzed and compared, and the sensitivity coefficient was recorded as $\mathrm{K}$.

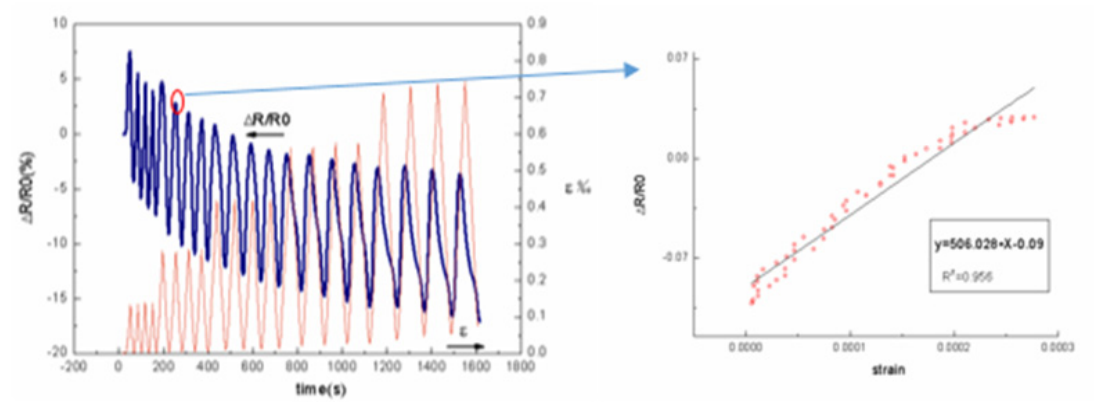

Fig.7 The piezoresistive effect curve of CFRCM2 at 28 day and the relationship between the change rate of cyclic resistance and strain in the second loading stage

The fig.7 shows that the piezoresistive effect of the specimen is negative at 28 day[14], and with the loading cycle, the piezoresistive effect curve shows a downward trend, which may be caused by polarization. In the first four cycles of loading, the linear variation of piezoresistive effect is good. When the loading reaches $25 \mathrm{kN}$, the corresponding piezoresistive effect curve changes nonlinearly. Especially in the unloading stage, this may be caused by the change of conductive network caused by the pore water being squeezed into the capillary hole under high stress, and not be recovered in time during unloading. The curve of resistivity change and strain change corresponding to this stage is shown on the right side of Fig. 6. According to the test fitting results, the slope of the linear change is the sensitivity coefficient K28 is 506.028 .

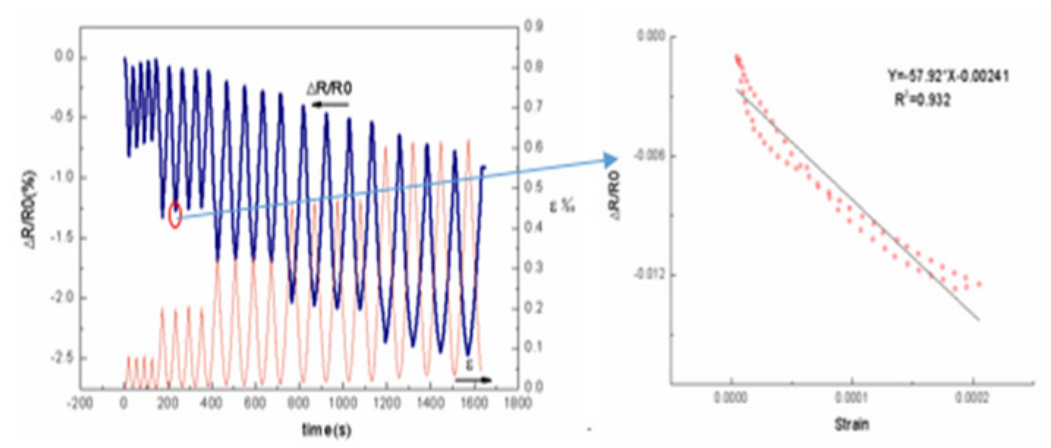

Fig.8 The piezoresistive effect curve of CFRCM2 at 90 day and the relationship between the change rate of cyclic resistance and strain in the second loading stage

From Fig.8, it can be seen that when the age is 90 days, the temperature is higher, and the moisture evaporation in the specimen is faster, and the piezoresistive effect curve of the specimen is a positive piezoresistive effect [14], With the increase of hydration and evaporation of water, the resistance of the specimen increases, and the internal ionic conductivity network decreases or the original connected conductive network is interrupted or partially interrupted. Under the action of stress, the conductivity of the specimen increases, and the original disconnected conductivity network reconnects, and the resistance decreases. Due to the existence of polarization, the piezoresistive effect curve still moves downward, but the piezoresistive effect is linear and reversible. The curve of the relationship between conductivity and strain at this stage is shown on the right side of Fig.8. According to the test fitting results, the slope of the linear change is the sensitivity coefficient K90 is -57.92 .

With the increase of age, the resistance value of the specimen increases continuously, and the piezoresistive effect curve also changes with the change of environmental humidity. When the age is 180 days, the temperature is higher. The piezoresistive effect curve and piezoresistive effect sensitivity coefficient are shown in Fig.9. Similar to 90 days of age, the piezoresistive effect was still positive, but the absolute value of sensitivity coefficient decreased, K180 is -24.213 . At this stage, the polarization effect tends to be stable at the end of the second loading cycle due to the small water content of the specimen, and the linear variation of piezoresistive effect is poor. Burr can be seen in the piezoresistive effect curve. This may be due to the continuous occurrence of microcracks in cement-based materials 
under the effect of stress gradient, and the conductivity

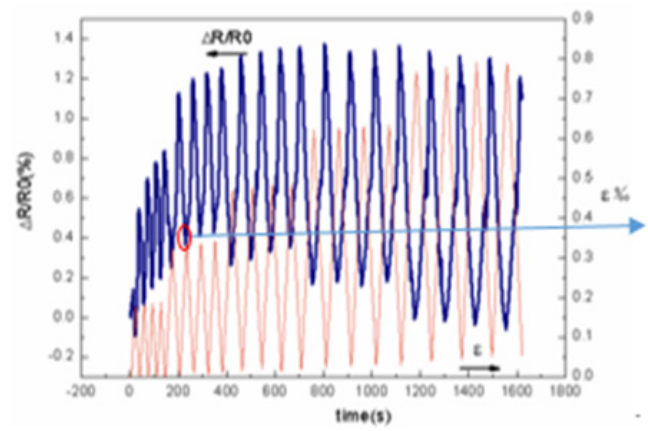

path cut by the microcracks can not be restored in time.

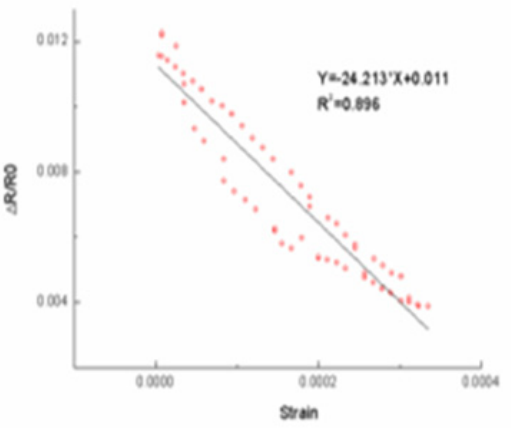

Fig.9 The piezoresistive effect curve of CFRCM2 at 180 day and the relationship between the change rate of cyclic resistance and strain in the second loading stage

When the age is 270 days, the temperature is the lowest and the corresponding environmental humidity is relatively low, but the evaporation desorption of the specimen is small, and the adsorption effect is dominant. With the increase of water adsorbed on the surface of the specimen, the specimen shows negative piezoresistive effect again under the action of force. It can be seen from figure 10 that the piezoresistive effect curve corresponding to this stage has good linearity and reversibility. Because the overall water content of the specimen is small and the polarization effect of electric field is small, the piezoresistive effect of the specimen is relatively stable. At this stage, the sensitivity coefficient of piezoresistive effect K270 is 1287.448 , and the change rate of resistance is close to linear change with strain. The stress-strain sensor can be made by sealing the corresponding specimen at this stage, and the related research needs to be further explored.
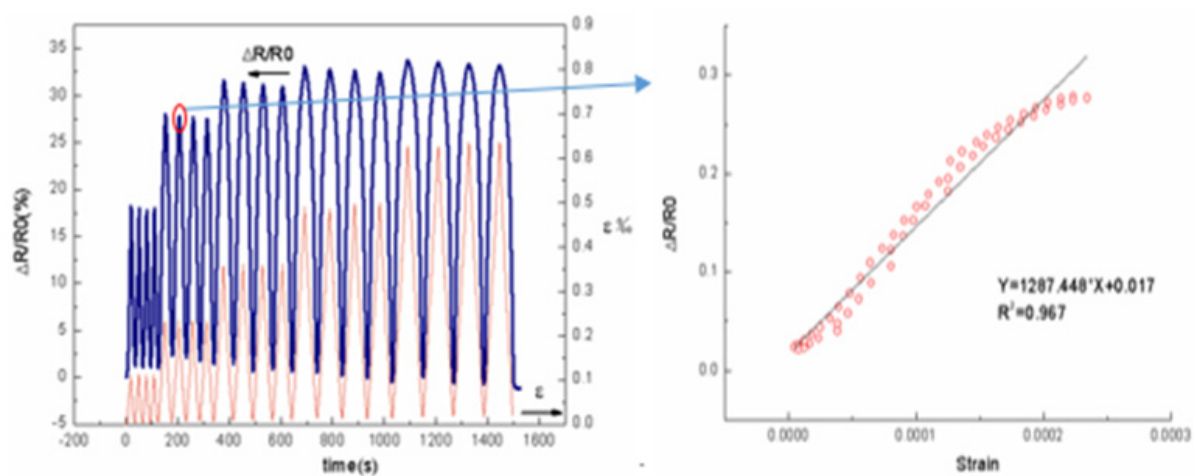

Fig.10 The piezoresistive effect curve of CFRCM2 at 270 day and the relationship between the change rate of cyclic resistance and strain in the second loading stage

As shown in Figure 11, when the age is 360 days, the ambient temperature and humidity all rise, but the adsorption effect of the specimen is still higher than that of desorption, and the piezoresistive effect still shows negative piezoresistive effect. The results show that the piezoresistive effect of the specimen is linear and reversible due to the lower polarization. The sensitivity coefficient K360 is 1439.51 . But in this stage, the peak value of piezoresistive effect changes " $\mathrm{W}$ " in high stress state, which may be caused by the complex changes of ionic conductance pathway and strain change in high strain state.

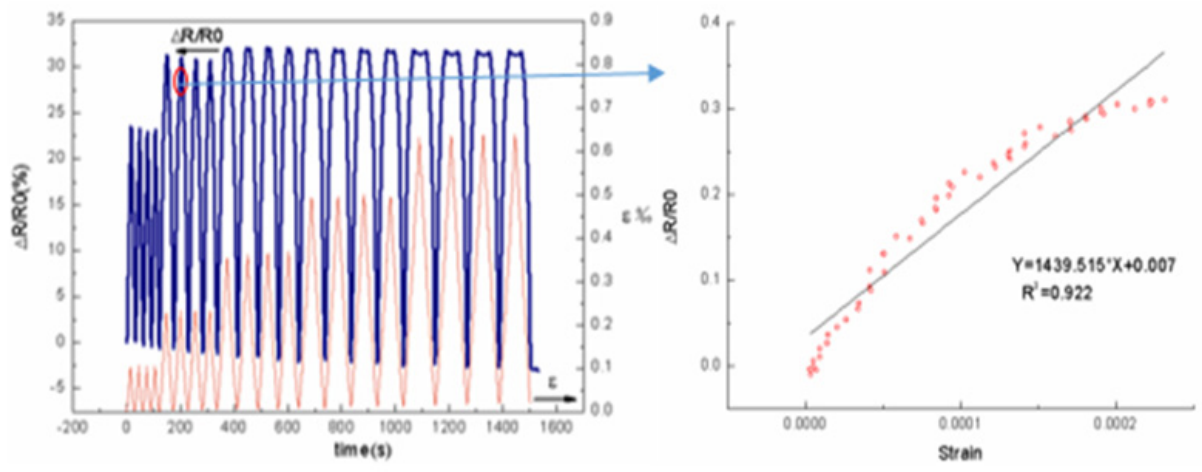

Fig.11 The piezoresistive effect curve of CFRCM2 at 360 day and the relationship between the change rate of cyclic resistance and strain in the second loading stage 


\section{Conclusions}

In this paper, the influence of age and humidity on the mechanical and electrical properties of carbon fiber reinforced cement mortar is discussed in detail. From that research, the following conclusions are drawn.

(1) When the age is small, the flexural strength of CFRCM is slightly higher than that of blank specimen. When the age was 180 days, the flexural strength of CFRCM1 was $11.21 \mathrm{MPa}$, which was $8.94 \%$ higher than that of CM; the flexural strength of CFRCM2 was 11.71 $\mathrm{MPa}, 13.8 \%$ higher than $\mathrm{CM}$. At the age of 270 days and 360 days, the flexural strength of CFRCM1 was 11.56 $\mathrm{MPa}$ and $11.82 \mathrm{MPa}$, which was $8.85 \%$ and $10.47 \%$ higher than that of CM; the flexural strength of CFRCM2 was $12.51 \mathrm{MPa}$ and $12.71 \mathrm{MPa}, 17.8 \%$ and $18.79 \%$ higher than $\mathrm{CM}$, respectively.

(2) The resistance of CFRCM increases with age, but the environmental temperature and humidity have significant effects on it. When adsorption is dominant, the resistance will decrease.

(3) The positive and negative conversion of the piezoresistive effect of carbon fiber cement mortar is closely related to the change of adsorbed water. CFRCM2 has higher sensitivity. When the age is 180 days, the sensitivity coefficient of piezoresistive effect is the smallest, K180 is - 24.213. When the age is 360 days, the sensitivity coefficient of piezoresistive effect is the largest, K360 is 1439.515.

\section{Acknowledgments.}

The authors acknowledge the financial support provided by the

Key Laboratory of Structure and Wind Tunnel of Guangdong Higher Education Institutes (201601 and 201803)

\section{References}

1. Chen, P.W., Chung, D.D.L. (1993) Carbon fiber reinforced concrete for smart structures capable of non-destructive flaw detection. Smart. Mater. Struct., 2: 22-30.

2. Xie, P., Gu, P., Beaudoin, J.J. (1996) Electrical percolation phenomenon in cement composites containing conductive fibers. J. Mater. Sci. 31: 4093-7.

3. Wang, X., Fu, X., Chung, D.D.L. (1999) Strain sensing using carbon fiber. J. Mater. Res. 14: 790-802.

4. Wen, S., Chung, D.D.L. (2001) Uniaxial compression in carbon fiber reinforced cement, sensed by electrical resistivity measurement in longitudinal and transverse directions. Cem. Concr. Res. 31:297-301.

5. Chen, B., Wu, K., Yao, W. (2004) Conductivity of carbon fiber reinforced cement-based Composites. Cem. Concr. Compos. 26:291-7.

6. Brandt, A.M. (2008) Fibre reinforced cement-based (FRC) composites after over 40 years of development in building and civil engineering. Compos. Struct. 86:3-9.

7. Hossain, M.Z., Awal, A.S.M.A. (2011) Flexural response of hybrid carbon fiber thin cement composites. Constr. Build Mater. 25: 670-677.

8. Xu, J., Yao,W., Wang, R. (2011) Nonlinear conduction in carbon fiber reinforced cement Mortar. Cem. Concr. Compos. 33:444-448.

9. Sun, M.Q., Li, J., Wang,Y.J., et al. (2015) Preparation of carbon fiber reinforced cement-based composites using self-made carbon fiber mat. Constr. Build. Mater. 79:283-289.

10. Han, B.G., Ding,S., Yu, X. (2015) Intrinsic self-sensing concrete and structures: a review. Measurement. 59:110-128.

11. Han, B.G., Zhang, L.Q., Zhang, C.Y., et al. (2016) Reinforcement effect and mechanism of carbon fibers to mechanical and electrically conductive properties of cement-based materials. Constr. Build. Mater. 125:479-489.

12. Wen, S.H., Chung, D.D.L. (2006) The role of electronic and ionic conduction in the electrical conductivity of carbon fiber reinforced cement. Carbon. 44: 2130-2138.

13. Chung, D.D.L. (2001) Comparison of submicron-diameter carbon filaments and conventional carbon fibers as fillers in composite materials. Carbon. 39: 1119-1125.

14. Wang, Y.L., Zhao, X.H. (2005) Positive and negative pressure sensitivities of carbon fiber-reinforced cement-matrix composites and their mechanism. Acta. Mater. Compos. Sin. 22:40-46.

15. Wen, S.H., Chung, D.D.L. (2008) Effect of Moisture on Piezoresistivity of carbon fiber-reinforced cement paste. ACI. Mater. J. 105:274-280.

16. Garboczi, E.J. (1990) Permeability, diffusivity, and microstructural parameters: a critical review. Cem. Concr. Res. 20:591-601.

17. Sohn, D., Mason, T.O. (1998) Electrically induced microstructural changes in portland cement pastes. Advn. Cem. Bas. Mat. 7: 81-88.

18. Susanto, A., Koleva, D. A., Breugel, K.V. (2017) The effect of water to cement ratio and curing on material properties of mortar specimens in stray current conditions. J. Adv. Concr. Technol. 15:627-643. 